\title{
Human dermal fibroblasts support the development of human primordial/primary follicles in a 3-dimensional alginate matrix culture system
}

\author{
Yuanyuan Wu ${ }^{1 \#}$, Zili Sun ${ }^{1 \#}$, Yu Wang ${ }^{1}$, Hong Chen ${ }^{1}$, Jiang Bian ${ }^{2}$ \\ ${ }^{1}$ Reproductive Center, Shanghai First Maternity and Infant Hospital, Tongji University School of Medicine, Shanghai, China; ${ }^{2}$ Department of \\ Obstetrics and Gynecology, Shanghai Everjoy Medical Polyclinic, Shanghai, China \\ Contributions: (I) Conception and design: Y Wu, J Bian; (II) Administrative support: H Chen; (III) Provision of study materials or patients: None; \\ (IV) Collection and assembly of data: Y Wu, J Bian, Z Sun, Y Wang; (V) Data analysis and interpretation: Y Wu, J Bian; (VI) Manuscript writing: All \\ authors; (VII) Final approval of manuscript: All authors. \\ "These authors contributed equally to this work. \\ Correspondence to: Jiang Bian. Department of Obstetrics and Gynecology, Shanghai Everjoy Medical Polyclinic, 675 Minbei Road, Shanghai 201107, \\ China. Email: bianjiang07110924@163.com; Hong Chen. Reproductive Center, Shanghai First Maternity and Infant Hospital, Tongji University \\ School of Medicine, Shanghai 210204, China. Email: chenhong@51mch.com.
}

Background: Alginate matrix 3-dimensional culture offers the opportunity for the development and maturation of human secondary follicles in vitro. However, alginate may not be the most suitable culture system for human primordial/primary follicles in vitro. Thus, the innovation of alginate matrix 3-dimensional culture systems for human primordial/primary follicles could hold promise as an ideal approach to restoring fertility.

Methods: We extracted primordial/primary follicles from ovarian tissues collected from patients with nonovarian benign gynecological conditions. Fibroblasts were isolated from dermal tissue from 1 male patient who had undergone posthectomy. The isolated human follicles were randomly divided into 2 groups and encapsulated within fibroblast-alginate-hydrogels or alginate hydrogels. The survival and growth of human primordial/primary follicles were measured after 21 days of in vitro culture.

Results: The dermal fibroblasts in alginate hydrogel microcapsules were round in shape, and were distributed as uniform clouds on the surface and gaps of the alginate. After 21 days of culture, the survival rate of follicles in the fibroblast-alginate group was higher than that of the alginate group $(\mathrm{P}<0.05)$. The diameter of follicles in the fibroblast-alginate group and the alginate group after 21 days of culture was $152.80 \pm 13.64$ and $129.14 \pm 9.95 \mu \mathrm{m}$, respectively $(\mathrm{P}<0.05)$. After 21 -day culture, the mean cpm (log-converted) for $3 \mathrm{H}$-thymidine incorporated by granulosa cells in the fibroblast-alginate and alginate groups was $6.87 \pm 0.24$ and $4.63 \pm 0.38$, respectively $(\mathrm{P}<0.05)$. After 21 days of culture, the messenger RNA expression levels of growth differentiation factor 9 (GDF9) and bone morphogenetic protein 15 (BMP15) were significantly higher in oocytes in fibroblast-alginate hydrogels than in those in alginate hydrogels $(\mathrm{P}<0.05)$.

Conclusions: Human fibroblasts are beneficial to the development of human follicles in 3-dimensional culture alginate gel systems over a long period of time. More studies are required to investigate the molecular biological mechanisms of human fibroblasts that promote follicle growth in vitro.

Keywords: Human; dermal fibroblasts; ovarian follicle; alginate; in vitro culture

Submitted Feb 26, 2021. Accepted for publication May 19, 2021.

doi: $10.21037 / \mathrm{atm}-21-2125$

View this article at: http://dx.doi.org/10.21037/atm-21-2125 


\section{Introduction}

Major advances in chemotherapy and radiotherapy have resulted in a marked improvement in the survival of young girls and women with cancer over the last decade (1). However, these treatments can put recipients at risk of losing their fertility $(2,3)$. Ovarian cryopreservation and reimplantation studies in humans initially suggested the possibility of restoring follicular activity to some degree (4). However, ovarian tissue transplantation is associated with the risk of malignant disease transmission from cryopreserved tissue (5).

In recent years, the promise of in vitro growth of human pre-antral follicles as an option for safeguarding the fertility of cancer patients who face the risk of premature ovarian failure has been reported (6). To date, 2-dimensional system culture of isolated pre-antral human follicles has failed to yield adequate outcomes (7-11). However, recently, alginate hydrogel 3-dimensional (3D) systems have achieved success in the in vitro culture of pre-antral follicles from mice (12-16) and rats (17). Also, isolated bovine pre-antral follicles (after vitrification and warming) cultured using an alginate 3D system have been shown to have higher viability and a higher rate of normal morphology (18). Novel tyraminebased hyaluronan (HA) hydrogel is especially appealing as a $3 \mathrm{D}$ model for the cultivation of ovarian follicles, owing to its ability to encapsulate pre-antral follicles from mice with ease, together with its visible transparency, moldability, and contribution to follicular growth, estradiol secretion, and meiosis resumption (19). Non-human primate and human secondary follicles isolated from ovarian tissue have previously been successfully cultivated in calcium alginate hydrogels (20-24). Alginate has a strong affinity with water and, when calcium ions are present, is able to form gels under mild conditions; consequently, it is among the most commonly applied biomaterials for microencapsulation (25-29). However, alginate alone without well-defined signals (i.e., extracellular matrix or growth factors) does not interact with integrins of mammalian cells or regulate somatic cell growth and development $(30,31)$, which is not conducive to the development of isolated human primordial/primary follicles (24). In a previous study, many putative local regulators (including extracellular matrix and cytokines) were secreted via autocrine and paracrine signaling between follicles and ovarian stromal cells, which appeared to influence the development of human primordial/primary follicles $(6,32,33)$. For instance, growth differentiation factor 9 (GDF9) and bone morphogenetic protein 15 (BMP15), which are secreted by oocytes, are believed to play critical synergistic roles in the proliferation and development of granulosa cells (24).

Therefore, the effective and safe supplementation of extracellular matrix and cytokines in alginate gel to simulate the development pattern of follicles in vivo is a crucial step in ameliorating the in vitro culture microenvironment of isolated human follicles. Recently, theca-interstitial cells (TICs) (34) and mouse embryonic fibroblasts (MEFs) (35) were used to enhance mouse follicle survival and growth in vitro, which can secrete extracellular matrix and cytokines in in vitro culture. These studies suggested that co-culture $3 \mathrm{D}$ systems may be beneficial for the development of follicles. As feeder cells, human dermal fibroblasts (HDFs) secrete extracellular matrix and cytokines, which have the effects of enriching the medium, adhering to the extracellular matrix, or interacting with membrane-bound proteins, thus supporting the successful development of human embryonic stem cells and embryos in vitro (36). In the present study, we primarily focused on exploring the distribution and viability of HDFs in alginate scaffold microcapsules, and also attempted to investigate the effects of fibroblasts in calcium alginate scaffold on human primordial/primary follicular growth and survival in vitro.

We present the following article in accordance with the MDAR reporting checklist (available at http://dx.doi. org/10.21037/atm-21-2125).

\section{Methods}

\section{Human ovarian tissue collection}

The present study was approved by the Ethics Committee of the First Maternity and Infant Hospital Affiliated to Tongji University (No. TJLAC-018-062). All procedures performed in this study involving human participants were in accordance with the Declaration of Helsinki (as revised in 2013). Strips of ovarian tissue were obtained from 34 women (aged 23 to 37 years) with benign gynecological diseases by laparoscopic surgery or laparotomy. Dermal tissue was obtained from 1 male patient (aged 24 years) who had undergone posthectomy. Ovarian and dermal tissue specimens incubated with HEPES-buffered modified Eagle medium were placed on ice and sent to the laboratory. Written informed consent was obtained from every participant in the study.

\section{Freezing/thawing of the ovarian tissues}

The ovarian tissue strips were frozen following a method 
detailed previously (37) with some modifications. Firstly, tissues were suspended in a tube containing $1 \mathrm{~mL}$ minimal essential medium (MEM), which was subsequently replaced with an identical quantity of cryoprotective solution with $10 \%$ dimethyl sulfoxide (DMSO) Sigma, St Louis, MO, USA) and 2\% human serum albumin (Sanquin, Amsterdam, the Netherlands) in MEM (Gibco) at $0{ }^{\circ} \mathrm{C}$. The cryotube was frozen using a programmable freezer according to the following steps: cooling from 0 to $-8{ }^{\circ} \mathrm{C}\left(-2{ }^{\circ} \mathrm{C} /\right.$ minute $)$, seeding via touching the cryovials with forceps pre-chilled with liquid nitrogen, cooling to $-150{ }^{\circ} \mathrm{C}\left(-0.3{ }^{\circ} \mathrm{C} /\right.$ minute $)$, and transfer to a liquid nitrogen tank. The ovarian tissue specimens were thawed by exposing the cryovials to room temperature for 2 minutes and immersing them in a water bath at $37^{\circ} \mathrm{C}$ to completely melt the ice. The ovarian tissues were transferred to MEM-containing Petri dishes and subjected to washing 3 times to isolate follicles.

\section{Ovarian follicle isolation}

The isolation of follicles from ovarian tissue was performed using a procedure described in a previous study (38). Ovarian tissue was serially cut into small fragments measuring $1 \mathrm{~mm}$ in width using the McIlwain Tissue Chopper (The Mickle Laboratory, Guildford, UK). Then, we placed the fragments into $50-\mathrm{mL}$ tubes with $0.07 \mathrm{mg} / \mathrm{mL}$ Liberase enzyme (Roche, Indianapolis, USA) and $20 \mathrm{U} / \mathrm{mL}$ DNA enzyme (Sigma-Aldrich Co. Carlsbad, CA, USA) before incubating the tubes in a water bath at $37^{\circ} \mathrm{C}$ for 1 hour. The tube contents were subjected to blowing and aspiration with Pasteur pipettes at 15-minute intervals. An equal volume of MEM (with $10 \%$ human serum albumin) and centrifugation at $50 \mathrm{~g}$ for 10 minutes at $4{ }^{\circ} \mathrm{C}$ were used to terminate digestion. The pellets were transferred to culture dishes, and a stereomicroscope was used to investigate the follicles (Leica, Wetzlar, Germany). Dulbecco's Phosphate-Buffered Saline (D-PBS) with $10 \%$ human serum albumin was used to wash the follicles after isolation.

\section{Proliferation and isolation of HDFs}

HDFs were isolated from skin specimens according to the previously described protocol (39). Briefly, samples of subcutaneous adipose tissue were removed and cut into $2 \mathrm{~mm} \times 2 \mathrm{~mm}$ strips using Dispase II (Roche, Penzberg, Germany). After the careful removal of the epidermal layer, the dermis was minced and subjected to 3 hours' incubation under magnetic rotation with $0.1 \%$ collagenase I (Roche, Penzberg, Germany). A mesh stainless steel mesh filter (BD biosciences, Hamburg, Germany) was used to filter the mixture, after which the filtrate was centrifuged (10 minutes at $100 \times \mathrm{g}$ ) 3 times with Dulbecco's Modified Eagle Medium (DMEM, $0.15 \%$ human serum albumin) for washing. A hemocytometer was employed for cell counting. Cell viability exceeded $95 \%$, as determined by $0.5 \%$ Trypan Blue exclusion.

Fibroblasts were plated separately at $2 \times 10^{6} / \mathrm{mL}$ in a $25-\mathrm{cm}^{2}$ culture flask (Nunc Inc., Roskilde, Denmark) at $37.0{ }^{\circ} \mathrm{C}$ in a humidified atmosphere of $5 \% \mathrm{CO} 2$ in air. Medium changes were conducted at 2-day intervals until confluency had been reached, at which point, the cells were rinsed 2 times with phosphate-buffered saline (PBS) without $\mathrm{Ca}^{2+}$ and $\mathrm{Mg}^{2+}$. For fibroblast collection, cells were subjected to incubation in $0.02 \%$ trypsin PBS and ethylenediamine tetraacetate dihydrate (EDTA) at $37.0{ }^{\circ} \mathrm{C}$ for 2 to 4 minutes followed by centrifugation $(10$ minutes at $100 \times \mathrm{g})$ with DMEM 3 times for washing. The cell sediment was suspended in $1 \mathrm{ml}$ of the frozen solution (10\% DMSO10\% FBS + 90\% DMEM) in cryovials. The cryovials were placed on the surface of liquid nitrogen for 30 minutes, and then quickly put into liquid nitrogen. We thawed the cryovials by immersing them in a water bath at $37^{\circ} \mathrm{C}$ for 3 minutes until all the ice had melted. Then, for washing, the frozen cell suspension was centrifuged $(10$ minutes at $100 \times \mathrm{g})$ with DMEM 3 times.

\section{Embedding and in vitro culture of follicles}

Follicles were randomly divided into the alginate group (AG) and the fibroblast-alginate group (FAG) (sodium alginate 55-65\% guluronic acid, FMC BioPolymers Philadelphia, $\mathrm{PA})$. Culture was conducted using a procedure previously reported (24) with modifications, as follows: Isolated single follicles were transferred with a stripper tip to droplets $(6 \mu \mathrm{L})$ of alginate hydrogel solution $(1.5 \% \mathrm{w} / \mathrm{v})$ or $\mathrm{HDF}$ alginate hydrogel solution [HDF concentration $\left(5 \times 10^{4} / \mathrm{mL}\right)$ in $1.5 \%(\mathrm{w} / \mathrm{v})$ alginate hydrogel]. The droplets were slowly released into a small beaker containing a $\mathrm{CaCl} 2(0.1 \mathrm{M})$ solution. Then, the beads in which the follicles were contained were removed from the beaker and subjected to 3 washes with MEM.

We grew the follicles in 96-well plates (with 1 follicle per well) in $100 \mu \mathrm{L}$ culture medium comprising MEM supplemented with $10 \%$ human serum albumin, $0.47 \mathrm{mM}$ pyruvic acid (Sigma), $50 \mathrm{IU} / \mathrm{mL}$ penicillin (Sigma), and 50 
$\mathrm{mg} / \mathrm{mL}$ streptomycin sulfate (Sigma) with $5 \% \mathrm{CO}_{2}$ at $37^{\circ} \mathrm{C}$. The addition of insulin, transferrin, and selenium (ITS-G; Gibco) was also made to the medium $(10 \mathrm{mg}$ insulin $/ \mathrm{mL}$, $5.5 \mathrm{mg}$ transferrin/mL, and $6.7 \mathrm{ng}$ sodium selenite $/ \mathrm{mL}$ ). At 2-day intervals, $50 \%$ of the culture media was replaced. All follicles were photographed with a Leica DM IL light microscope (Leica, Wetzlar, Germany) equipped with phase objectives, a heated stage, and a Spot Insight 2 Megapixel Color Mosaic camera and Spot software (Spot Diagnostic Instruments, Sterling Heights, MI, USA). After that, ImageJ (National Institutes of Health, USA) was used to measure the follicular diameter. Oocyte diameters were measured without zona pellucida.

\section{Frozen sections and scanning electron microscopy of HDF- alginate hydrogels}

The beads in the FAG were coated with OCT (a watersoluble polyethylene glycol and polyvinyl alcohol mixture) embedding medium for frozen head. The cold head was quickly put in a LEICA CM1950-type frozen section machine (Leica Mikrosysteme Vertrieb GmbH Leica Wetzlar, 35578 Germany), frozen on stage at $-25^{\circ} \mathrm{C}$ for 3 minutes, and was frozen sections, cold dry, 10\% neutral formaldehyde 40 seconds, hematoxylin-eosin (HE) staining, dehydration, cementing. Fibroblasts were observed under a microscope. The beads in the FAG were also fixed in 2.5\% glutaraldehyde for 48 hours and dehydrated in gradient volume fractions of ethanol $(0.70,0.85,0.95$, and 1.00), which was followed by critical point drying and vacuum coating with pure gold. Fibroblast distribution was observed with an SM-T300 scanning electron microscope (SEM) (JEOL Technics Co., Ltd., Tokyo, Japan).

\section{Detection of follicular activity}

The viability of follicles was assessed as described previously (40). We subjected follicles to incubation in D-PBS containing $2 \mathrm{mmol} / \mathrm{L}$ calcein-AM and $5 \mathrm{mmol} / \mathrm{L}$ ethidium homodimer-I for 20 minutes at $37{ }^{\circ} \mathrm{C}$ in darkness. After washing in PBS, the follicles were examined using an inverted fluorescence microscope (DMIRB Leica Germany). In live cells, esterases cleave non-fluorescent cell-permeant calcein$\mathrm{AM}$ after its entry into the cell, resulting in the production of calcein, which is well retained in living cells and lends itself to an intense uniform green fluorescence (ex/em 495/515 nm). Ethidium homodimer-I binds to DNA with high affinity in cells with membrane damage, leading to a 40 -fold enhancement in fluorescence and the production of bright red fluorescence (ex/em 495/635 nm) in dead cells.

\section{H-thymidine incorporation capability of granulosa cells}

Culture medium was replaced with medium supplemented with 0.4-Ci methyl-3H-thymidine (PerkinElmer, Boston, USA; $1 \mu \mathrm{ci} / 100 \mu \mathrm{L}$ ) per well. Sixteen hours later, 5 follicles isolated from the FAG or AG were collected for each replicate and subjected to 2 washes with D-PBS, followed by dissolvement in $10 \mathrm{mM}$ EDTA. Next, a $3 \mathrm{H}$-thymidine incorporation assay was performed.

\section{Nested quantitative real-time PCR}

Follicles were removed from the alginate beads using insulin gauge needles. Follicles were transferred to culture dishes with $0.04 \mathrm{mg} / \mathrm{mL}$ Liberase enzyme (Roche, Indianapolis, USA) for 15 minutes, and mechanical separation of individual oocytes was performed via repeated aspiration using a narrow-bore Pasteur pipette under a stereomicroscope (Leica, Wetzlar, Germany). The gene expression of BMP15 and GDF9 in oocytes of FAG and AG were detected by Nested Quantitative Real-Time PCR assay as previously reported (41). Total RNA was extracted and reverse transcribed into complementary DNA, which was then amplified; finally, quantitative real-time PCR was carried out with Tagman probes (Shanghai Shinegene Molecular Biotechnology Co., Ltd.). Cycle threshold (CT) values indicating the quantity of the target gene in each sample were obtained, and target gene sequence determination was carried out in real time with the ABI Prism 7000 sequence detection system (Applied Biosystem, Foster City, CA). For each sample, the abundances of GDF9 and BMP15 messenger RNA (mRNA) were determined, with $\beta$-actin mRNA serving for normalization. For each sample, 3 replicates were performed, with the mean value taken for statistical analysis. Relative gene expressions were calculated according to the abundance ratio of each target gene to $\beta$-actin.

\section{Statistical analysis}

SPSS 11.5 (SPSS Inc.) was employed for all statistical analyses. Follicle viability was compared by performing the chi-square test. Follicular diameters were expressed as means \pm standard deviations, with the paired-sample $t$-test applied for comparisons. The Mann-Whitney $U$ test was used to compare BMP15 and GDF9 mRNA expression 

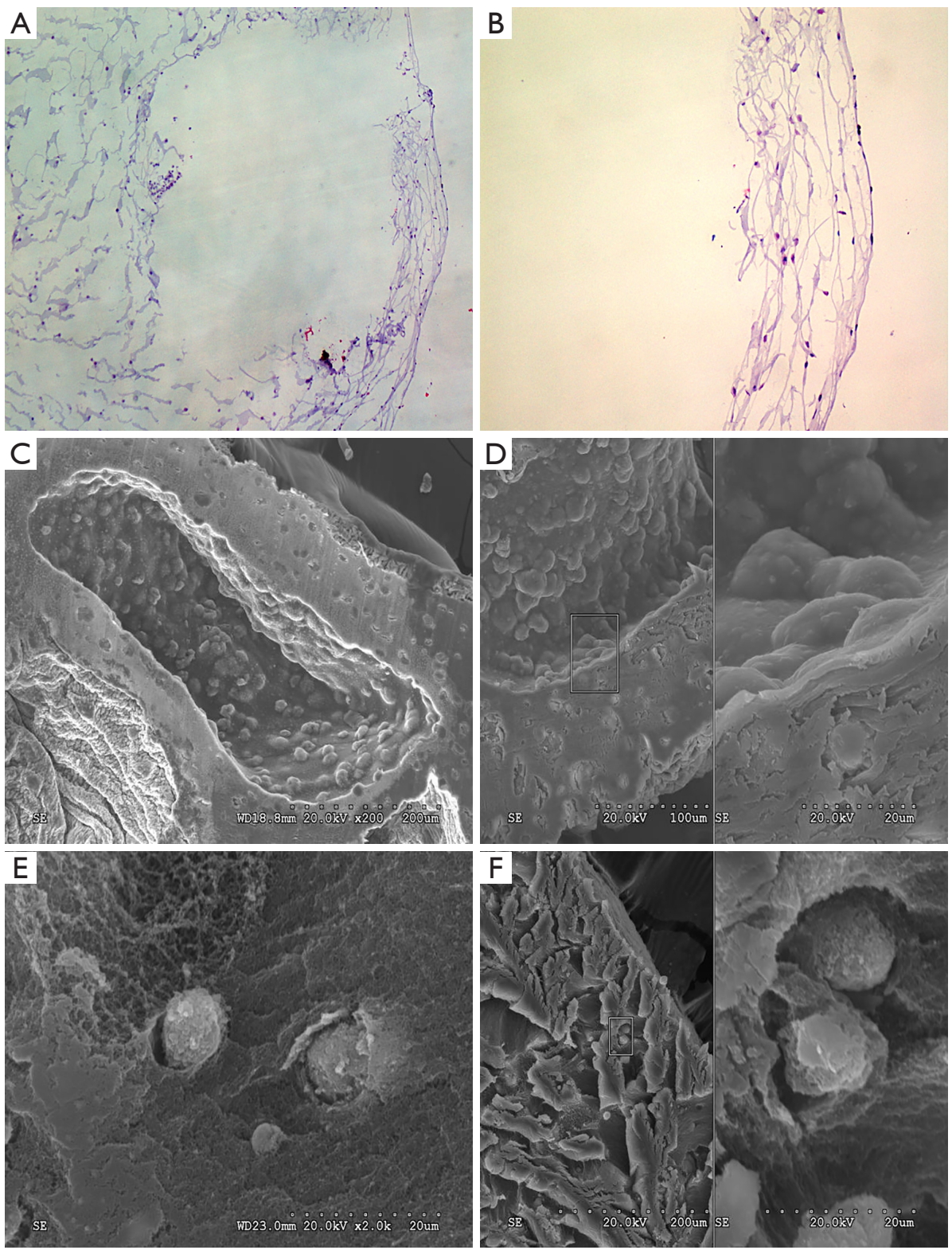

Figure 1 Characterization of fibroblasts in alginate matrix. Light microscope: $(A) \times 50,(B) \times 100$, after encapsulation and culture, fibroblasts (FC) were round, uniform clouds on the surface and in the gaps of the alginate; scanning electron microscope: $(\mathrm{C}, \mathrm{D}, \mathrm{E}, \mathrm{F})$.

between the 2 groups. $\mathrm{P}<0.05$ was indicative of a statistically significant difference.

\section{Results}

HDFs support the development of follicles in $3 D$ culture system

The morphology of HDFs $\left(5 \times 10^{4} / \mathrm{mL}\right)$ in the FAG was studied by HE staining and SEM (Figure $1 A, B)$. Fibroblasts in the fibroblast-alginate matrices group were round in shape, and were distributed as uniform clouds on the surface and gaps in alginate matrices (Figure 1C,D,E,F). The viability of fibroblasts in alginate matrix after 21 days of culture is shown in Figure 2.

Follicles were randomly divided into the FAG $(\mathrm{n}=68)$ or AG ( $\mathrm{n}=63)$, and were cultured for 21 days (Figure 3). Initially, the follicle diameter in the FAG and AG did not 


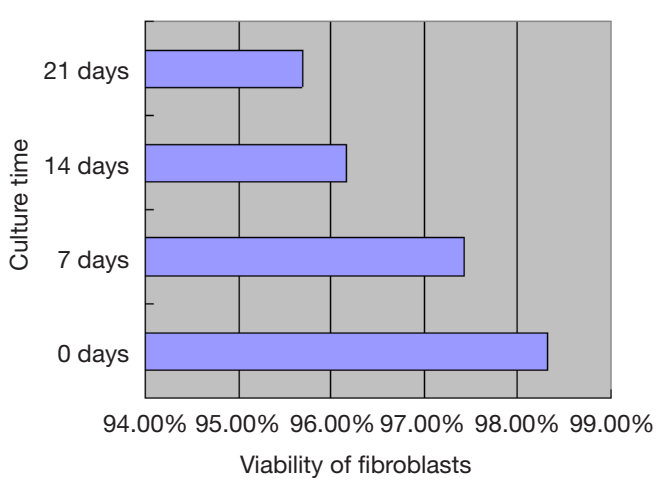

Figure 2 The viability of fibroblasts in alginate matrix in vitro after 21 days of culture. After 0, 7, 14, and 21 days of culture, the survival rate of fibroblasts in the fibroblast (FC)-alginate group did not show a statistically significant difference. $\mathrm{P}>0.05$.

show a significant difference $(\mathrm{P}>0.05)$. However, following 21 days' culture, follicles cultured in HDF-alginate matrices showed a significant increase in size compared to those cultured in alginate matrices, and the oocyte diameter in the FAG was significantly greater than that in the control group (Table 1). Follicle viability was assessed by calcein$\mathrm{AM}$ and ethidium homodimer-I in vitro culture, and the results are shown in Figure 4. After culture for 21 days, the survival rate of follicles in the FAG and AG was statistically significantly different (Figure 5). Alginate hydrogel with an HDF concentration of $5 \times 10^{4} / \mathrm{mL}$ effectively enhanced follicular survival.

\section{Proliferation of human follicular somatic cells}

A $[3 \mathrm{H}]$ thymidine incorporation assay was performed to assess human follicular somatic cell proliferation in the FAG and AG after 21 days culture. Following the completion of culture, incorporation of $\left[{ }^{3} \mathrm{H}\right]$ thymidine by follicles was observed under all media conditions, which indicated the occurrence of DNA replication and consequential cell proliferation. Also, $\left[{ }^{3} \mathrm{H}\right]$ thymidine incorporation was significantly increased in follicles subjected to co-culture with fibroblasts in alginate matrices in comparison with follicles cultured without fibroblasts (Table 2).

\section{The FAG had higher expression levels of GDF9 and BMP15 mRNA in oocytes}

To establish whether the expression levels of growth-related factors could reflect human follicle development, the oocyte-specific markers GDF9 and BMP15 were examined using real-time PCR after 21 days' culture, and comparisons were conducted between oocytes from the FAG and AG. After 21 days, the expressions of GDF9 and BMP15 mRNA in oocytes in the FAG were significantly higher than those in the $\mathrm{AG}(\mathrm{P}<0.05)($ Table 3$)$.

\section{Discussion}

As far as we are aware, the present study is the first to evidence that human primordial/primary follicles can grow in HDF-alginate matrices after 21 days' culture, which proves that HDFs in alginate matrix are a suitable support for in vitro long-term culture of isolated human primordial/ primary follicles.

The development of technologies to grow and mature oocytes from primordial/primary follicles holds much attraction for researchers studying fertility preservation (42). To date, researchers have created hydrogel encapsulationbased follicle culture systems with biomaterials, including alginate, which can maintain the spherical structure and cell-cell interactions of the follicles (22). In our study, follicles in the FAG showed substantial growth during the 21-day culture period, reaching a mean size of $150 \mu \mathrm{m}$. A previous study using an an alginate matrix culture system reported that follicles $(34-51 \mu \mathrm{m})$ derived from cryopreserved tissue survived for 7 days and grew to 44-70 $\mu \mathrm{m}$ (24). In the present study, the survival rate of primordial/primary follicles in the FAG was significantly higher than that of the control follicles after a relatively long culture period (21 days), showing that human foreskin fibroblasts had a significant effect on the survival of encapsulated follicles. When cultured within a $3 \mathrm{D}$ extracellular matrix such as collagen or fibrin, fibroblasts, as feeder cells, can secrete a richer, more complex extracellular matrix and cytokines, to influence other cellular functions in the in vitro culture environment (43). Various factors (including extracellular matrix, leukemia inhibitory factor (LIF), basic fibroblast growth factorb (FGF), kit ligand or stem cell factor, insulin-like growth factor, bone morphogenetic proteins, and activin A), which are secreted by fibroblasts, are critical in early follicle development (6). The results of the present study strongly suggest that extracellular matrix and cytokines secreted by fibroblasts in $3 \mathrm{D}$ alginate matrices may create a superior microenvironment for the growth of primordial/primary follicles. Therefore, unidirectional paracrine signaling from HDFs to follicles is seemingly the mechanism responsible 


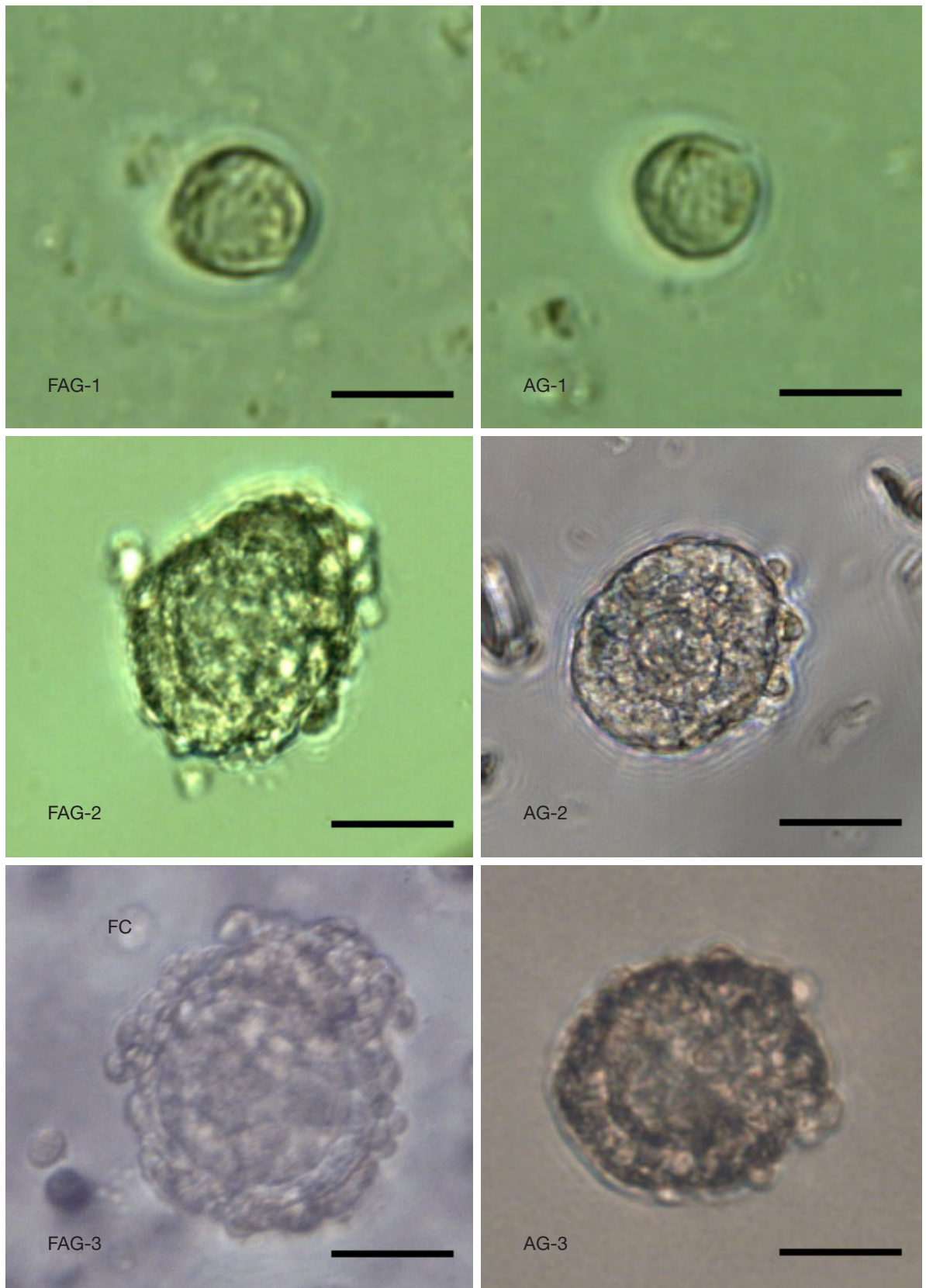

Figure 3 Scale bar: $100 \mu \mathrm{m}$. Follicles in the fibroblast (FC)-alginate group (FAG). Follicles in the alginate group (AG). FAG-1 and AG-1 after 7 days of culture; FAG-2 and AG-2 after 14 days of culture; FAG-3 and AG-3 after 21 days of culture. Follicles were examined for size measurements using an inverted Leica DM IRB microscope with transmitted light and phase objectives (Leica, Bannockburn, IL, USA).

for the stimulatory effect of HDFs on follicular growth. Nevertheless, this study did not attempt to identify the critical factors supporting and stimulating follicle growth by testing the extracellular matrix or cytokines secreted by HDFs. Therefore, more studies are required to investigate which types of HDF-secreted extracellular matrix or cytokines in 3D-alginate matrix are involved in regulating the initiation of human primordial/primary follicle growth during in vitro culture. A previous study also showed that the addition of low-dose 1,25-dihydroxy vitamin D3 (VD3) increased the survival of pre-antral follicles and maintained Anti-Mullerian hormone (AMH) production 
Table 1 Diameter of follicles cultured under different 3-dimensional culture systems for 21 days

\begin{tabular}{lllll}
\hline Group & No. of follicles & 7 days' culture & 14 days' culture & 21 days' culture \\
\hline AG & 63 & $64.43 \pm 9.68$ & $115.61 \pm 11.60$ & $129.14 \pm 9.95$ \\
FAG & 57 & $71.83 \pm 10.32$ & $120.42 \pm 8.90$ & $152.80 \pm 13.64^{*}$ \\
\hline
\end{tabular}

*follicles in the FAG were significantly larger than follicles in the AG after day 21 of culture. FAG, follicles embedded in fibroblast-alginate matrix; AG, follicles embedded in alginate matrix. Data are represented as the mean \pm SEM.
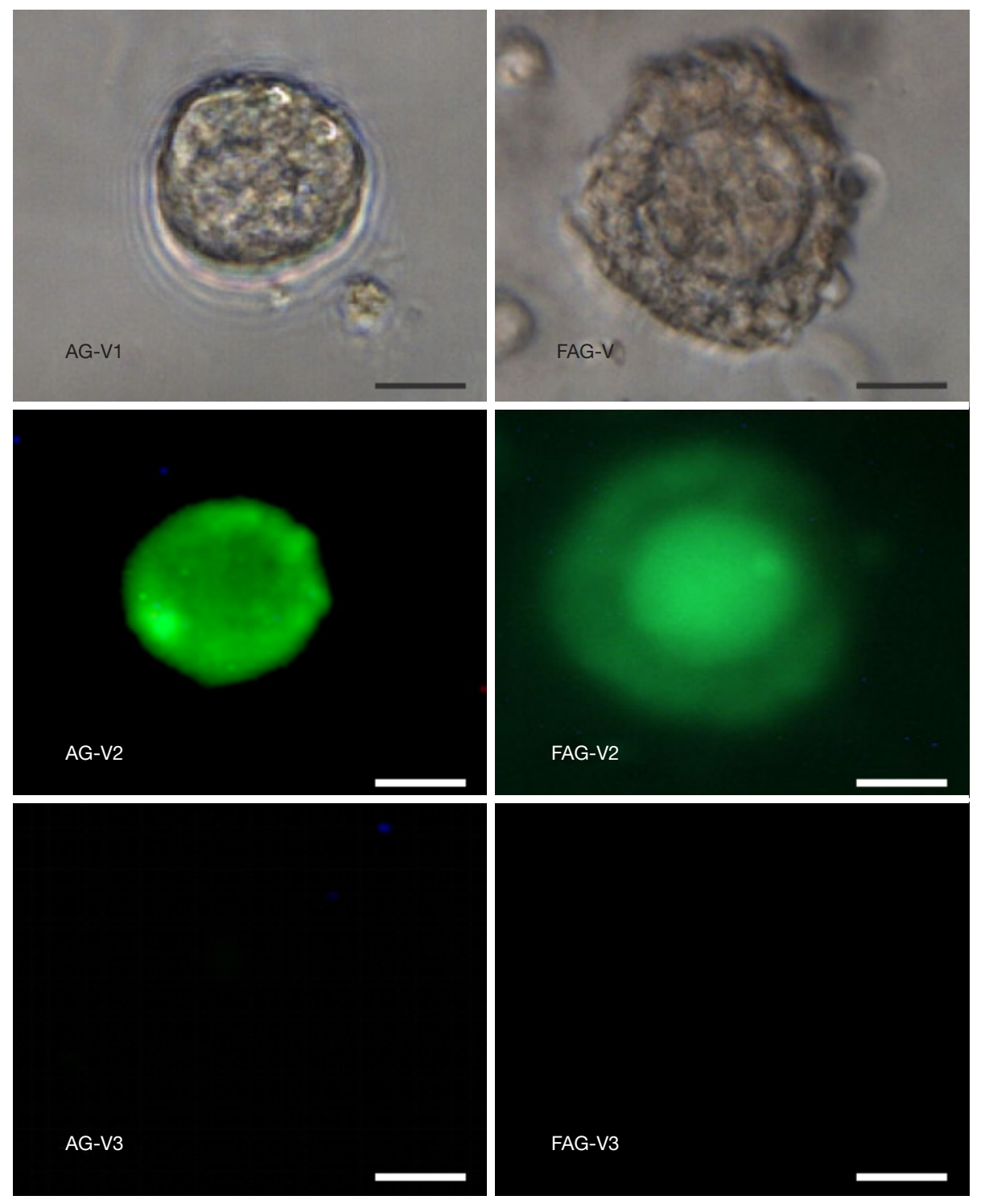

Figure 4 Follicular viability under 3D culture systems after 21 days of in vitro culture. The viability of follicles in the fibroblast-alginate group (FAG) and the alginate group (AG) after in vitro culture. The first line (FAG-V1, AG-V1) shows follicles under a light microscope. The second (FAG-V2, AG-V2) and third (FAG-V3, AG-V3) lines show follicular viability assessed by calcein-AM and ethidium homodimer-I (Leica inverted fluorescence microscope). Scale bar: $100 \mu \mathrm{m}$. 


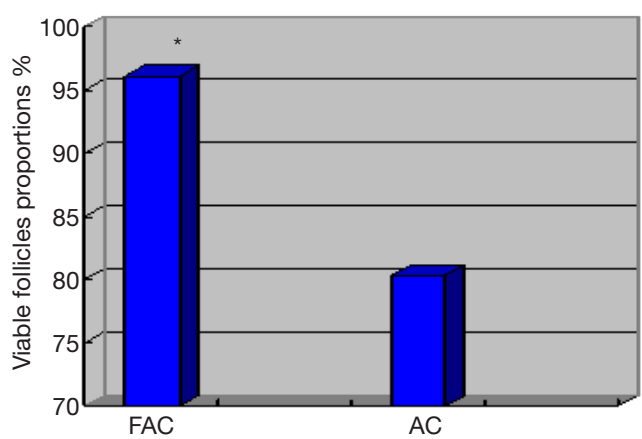

Figure 5 Proportions of viable follicles in the fibroblast-alginate group (FAG) and the alginate group (AG) after culture for 21 days. *the viability of follicles in the FAG $(96.17 \%)$ was significantly higher than that in the AG $(80.32 \%)(\mathrm{P}<0.05)$. FAG, follicles embedded in fibroblast-alginate matrix; AG, follicles embedded in alginate matrix.

Table 2 Comparison of cpm of $3 \mathrm{H}$-thymidine incorporation of granulosa cell follicles in the fibroblast-alginate group (FAG) and alginate group (AG) after culture for 21 days

\begin{tabular}{lc}
\hline Group & $\mathrm{cpm}$ \\
\hline AG & $4.63 \pm 0.38$ \\
FAG & $6.87 \pm 0.24^{\star}$
\end{tabular}

*follicular $3 \mathrm{H}$-thymidine incorporation in the FAG was significantly higher than that in the $A G(P<0.05)$. FAG, follicles embedded in fibroblast-alginate matrix; $A G$, follicles embedded in alginate matrix.

Table 3 Quantification PCR showed that the abundance of BMP15 and GDF9 mRNA in oocytes in the fibroblast-alginate group (FAG) was significantly higher than that in the alginate group (AG) after culture for 21 days

\begin{tabular}{lccc}
\hline Variable & FAG & AG & P value \\
\hline BMP15 & $39.64(6.60-92.08)^{\mathrm{a}}$ & $7.93(4.76-8.40)$ & $<0.05$ \\
mRNA & & & \\
GDF9 & $127.36(47.81-329.64)^{\mathrm{a}}$ & $41.42(4.96-224.06)$ & $<0.05$ \\
mRNA & & & \\
\hline
\end{tabular}

Data are expressed as medians with 25th-75th percentiles in parentheses, and comparisons were performed using the MannWhitney $U$ test. ${ }^{\mathrm{a}} \mathrm{P}<0.05$, compared with the FAG and AG.

by antral follicles, whereas high-dose VD3 improved antral follicle growth (44). In future, more studies are needed to reveal which factors can enhance the survival and growth capabilities of human pre-follicles encapsulated within human fibroblast-alginate matrix during culture.
There are reports of BMP15 and GDF9 expression in primordial as well as primary follicles (45-49) in humans at various developmental ages. Wang and Roy reported that recombinant GDF-9 derived from oocytes increased the numbers of primary and secondary follicles in ovarian cortical samples from humans and rodents in vitro, indicating it to play a critical part in the initiation and progression of follicular growth (50). Furthermore, BMP-15 has been shown to stimulate granulosa cell proliferation in a follicle stimulating hormone (FSH)-independent way (51). A previous study suggested that GDF9 and BMP15 expression in oocytes of patients with polycystic ovary syndrome could not be restored to a normal level, even by ovarian stimulation. In the same study, these factors were found to have abnormal expression patterns during oocyte maturation, which may be related to the impairment of oocyte quality and developmental competence in polycystic ovary syndrome (52). In the present study, the levels of GDF9 and BMP15 in oocytes in the FAG were significantly higher than those in the control group after 21 days' in vitro culture, which enabled the culture of human follicles within alginate hydrogels. However, the exact mechanism of how HDFs increase the expression of GDF9 and BMP15 in oocytes cultured in alginate hydrogels in vitro needs further investigation.

The results of this study show that the technologies of human primordial/primary follicles and HDFs in alginate gel co-culture systems may benefit young female patients with cancer who have undifferentiated status and large numbers of primordial follicles in helping to preserve their fertility. Theoretically speaking, the presence of ovarianderived fibroblasts may be conducive to the development of human follicles in vitro. However, the procedure of isolating and purifying ovarian-derived fibroblasts is more likely to be mixed with tumor cells. The advantages of isolating HDFs from healthy human derma are that they are homogenous, easy to produce, and commercially available (53). One of shortcomings of our research is that the HDFs were derived from male skin; in future, it is necessary to establish a fibroblast line derived from skin of a healthy female patient for the development of follicles in vitro.

In conclusion, our study has revealed that isolated human primordial/primary follicles encapsulated in human fibroblast-alginate matrix have greater capability to survive and grow during culture. However, it should be pointed out that the developmental competence of human oocytes from follicles was not examined in this study. Therefore, further studies are required to investigate the physiology of oocytes of human follicles in HDF-alginate matrices. 


\section{Acknowledgments}

Funding: This study was supported by funding from Scientific Research Project funded by Shanghai Municipal Commission of Health and Family Planning (No. 201740170).

\section{Footnote}

Reporting Checklist: The authors have completed the MDAR reporting checklist. Available at http://dx.doi.org/10.21037/ atm-21-2125

Data Sharing Statement: Available at http://dx.doi. org/10.21037/atm-21-2125

Conflicts of Interest: All authors have completed the ICMJE uniform disclosure form (available at http://dx.doi. org/10.21037/atm-21-2125). The authors have no conflicts of interest to declare.

Ethical Statement: The authors are accountable for all aspects of the work in ensuring that questions related to the accuracy or integrity of any part of the work are appropriately investigated and resolved. All procedures performed in this study involving human participants were in accordance with the Declaration of Helsinki (as revised in 2013). The present study was approved by the Ethics Committee of the First Maternity and Infant Hospital Affiliated to Tongji University (No. TJLAC-018-062). Written informed consent was obtained from every participant in the study.

Open Access Statement: This is an Open Access article distributed in accordance with the Creative Commons Attribution-NonCommercial-NoDerivs 4.0 International License (CC BY-NC-ND 4.0), which permits the noncommercial replication and distribution of the article with the strict proviso that no changes or edits are made and the original work is properly cited (including links to both the formal publication through the relevant DOI and the license). See: https://creativecommons.org/licenses/by-nc-nd/4.0/.

\section{References}

1. Donnez J, Martinez-Madrid B, Jadoul P, et al. Ovarian tissue cryopreservation and transplantation: a review. Hum Reprod Update 2006;12:519-35.
2. Demeestere I, Simon P, Emiliani S, et al. Orthotopic and heterotopic ovarian tissue transplantation. Hum Reprod Update 2009;15:649-65.

3. Picton HM, Kim SS, Gosden RG. Cryopreservation of gonadal tissue and cells. Br Med Bull 2000;56:603-15.

4. Dolmans MM, Donnez J, Camboni A, et al. IVF outcome in patients with orthotopically transplanted ovarian tissue. Hum Reprod 2009;24:2778-87.

5. Meirow D, Hardan I, Dor J, et al. Searching for evidence of disease and malignant cell contamination in ovarian tissue stored from hematologic cancer patients. Hum Reprod 2008;23:1007-13.

6. Picton HM, Harris SE, Muruvi W, et al. The in vitro growth and maturation of follicles. Reproduction 2008;136:703-15.

7. Roy SK, Treacy BJ. Isolation and long-term culture of human preantral follicles. Fertil Steril 1993;59:783-90.

8. Abir R, Roizman P, Fisch B, et al. Pilot study of isolated early human follicles cultured in collagen gels for 24 hours. Hum Reprod 1999;14:1299-301.

9. Abir R, Fisch B, Nitke S, et al. Morphological study of fully and partially isolated early human follicles. Fertil Steril 2001;75:141-46.

10. Hovatta O, Wright C, Krausz T, et al. Human primordial, primary and secondary ovarian follicles in longterm culture: effect of partial isolation. Hum Reprod 1999;14:2519-24.

11. Telfer EE, McLaughlin M, Ding C, et al. A two-step serum-free culture system supports development of human oocytes from primordial follicles in the presence of activin. Hum Reprod 2008;23:1151-58.

12. Pangas SA, Saudye H, Shea LD, et al. Novel approach for the three-dimensional culture of granulosa cell-oocyte complexes. Tissue Eng 2003;9:1013-21.

13. Kreeger PK, Fernandes NN, Woodruff TK, et al. Regulation of mouse follicle development by folliclestimulating hormone in a three-dimensional in vitro culture system is dependent on follicle stage and dose. Biol Reprod 2005;73:942-50.

14. Kreeger PK, Deck JW, Woodruff TK, et al. The in vitro regulation of ovarian follicle development using alginateextracellular matrix gels. Biomaterials 2006;27:714-23.

15. Xu M, Kreeger PK, Shea LD, et al. Tissue-engineered follicles produce live, fertile offspring. Tissue Eng 2006;12:2739-46.

16. Xu M, West $\mathrm{E}$, Shea $\mathrm{LD}$, et al. Identification of a stagespecific permissive in vitro culture environment for follicle growth and oocyte development. Biol Reprod 
2006;75:916-23.

17. Heise M, Koepsel R, Russell AJ, et al. Calcium alginate microencapsulation of ovarian follicles impacts FSH delivery and follicle morphology. Reprod Biol Endocrinol 2005;3:47.

18. Bus A, van Hoeck V, Langbeen A, et al. Effects of vitrification on the viability of alginate encapsulated isolated bovine pre-antral follicles. J Assist Reprod Genet 2018;35:1187-99.

19. Desai N, Abdelhafez F, Calabro A, et al. Three dimensional culture of fresh and vitrified mouse pre-antral follicles in a hyaluronan-based hydrogel: a preliminary investigation of a novel biomaterial for in vitro follicle maturation. Reprod Biol Endocrinol 2012;10:29-5.

20. Xu M, Barrett SL, West-Farrell E, et al. In vitro grown human ovarian follicles from cancer patients support oocyte growth. Hum Reprod 2009;24:2531-40.

21. Xu, M, West-Farrell, ER, Stouffer, RL, et al. Encapsulated three-dimensional culture supports development of nonhuman primate secondary follicles. Biol Reprod 2009;81:587-94.

22. Xu J, Bernuci MP, Lawson MS, et al. Survival, growth, and maturation of secondary follicles from prepubertal, young, and older adult rhesus monkeys during encapsulated threedimensional culture: effects of gonadotropins and insulin. Reproduction 2010;140:685-97.

23. Xu J, Lawson MS, Yeoman RR, et al. Secondary follicle growth and oocyte maturation during encapsulated three-dimensional culture in rhesus monkeys: effects of gonadotrophins, oxygen and fetuin. Hum Reprod 2011;26:1061-72.

24. Amorim CA, Van Langendonckt A, David A, et al. Survival of human pre-antral follicles after cryopreservation of ovarian tissue, follicular isolation and in vitro culture in a calcium alginate matrix. Hum Reprod 2009;24:92-9.

25. Dulieu C, Poncelet D, Neufeld R. Encapsulation and Immobilization Techniques. Cell Encapsulation Technology and Therapeutics 1999;10:3-17.</jrn>

26. Smidsrod O, Skjak-Braek G. Alginate as Immobilization Matrix for Cells. Trends Biotechnol 1990;8:71-8.

27. Amsden B, Turner N. Diffusion Characteristics of Calcium Alginate Gels. Biotechnol Bioeng 1999;65:605-10.

28. Martinsen A, Skjak-Braek G, Smidsrod O. Alginate as Immobilization Material: I. Correlation Between Chemical and Physical Properties of Alginate Gel Beads. Biotechnol Bioeng 1989;89:79-83.

29. Martinsen A, Storrø I, Skjårk-Braek G. Alginate as Immobilization Material: III. Diffusional Properties.
Biotechnol Bioeng 1992;39:186-94.

30. Haug A, Larsen B, Smidsrod O. Studies of the sequence of uronic acid residues in alginic acid. Acta Chemica Scandinavica 1967;21:691-704.</jrn>

31. Rowley JA, Madlambayan G, Mooney DJ. Alginate hydrogels as synthetic extracellular matrix materials. Biomaterials 1999;20:45-53.

32. Scott JE, Carlsson IB, Bavister BD, et al. Human ovarian tissue cultures: extracellular matrix composition, coating density and tissue dimensions. Reprod Biomed Online 2004;9:287-93.

33. Tingen CM, Kiesewetter SE, Jozefik J, et al. A macrophage and theca cell-enriched stromal cell population influences growth and survival of immature murine follicles in vitro. Reproduction 2011;141:809-20.

34. Tagler D, Tu T, Smith RM, et al. Embryonic fibroblasts enable the culture of primary ovarian follicles within alginate hydrogels. Tissue Eng Part A 2012;18:1229-38.

35. Hongisto H, Vuoristo S, Mikhailova A, et al. Laminin-511 expression is associated with the functionality of feeder cells in human embryonic stem cell culture. Stem Cell Res 2012;8:97-108.

36. Dolmans MM, Michaux N, Camboni A, et al. Evaluation of Liberase, a purified enzyme blend, for the isolation of human primordial and primary ovarian follicles. Hum Reprod 2006;21:413-20.

37. Zhang Z, Slobodianski A, Ito WD, et al. Enhanced collateral growth by double transplantation of genenucleofected fibroblasts in ischemic hind limb of rats. PLoS One 2011;6:e19192.

38. Cortvrindt RG, Smitz JE. Fluorescent probes allow rapid and precise recording of follicle density and staging in human ovarian cortical biopsy samples. Fertil Steril 2001;75:588-93.

39. Desai N, Alex A, AbdelHafez F, et al. Three-dimensional in vitro follicle growth: overview of culture models, biomaterials, design parameters and future directions. Reprod Biol Endocrinol 2010;8:119-25.

40. Rhee S. Fibroblasts in three dimensional matrices: cell migration and matrix remodeling. Exp Mol Med 2009;41:858-65.

41. Jing Xu, Jon D, David B. Direct vitamin D3 actions on rhesus macaque follicles in three-dimensional culture: assessment of follicle survival, growth, steroid and anti-Müllerian hormone production. Fertil Steril 2016;106:1815-20.

42. Margulis S, Abir R, Felz C, et al. Bone morphogenetic protein 15 expression in human ovaries from fetuses, girls 
and women. Fertil Steril 2009;92:1666-73.

43. Oron G, Fisch B, Ao A, et al. Expression of growthdifferentiating factor 9 and its type-I receptor in human ovaries. Reprod Biomed Online 2010;21:109-17.

44. Aaltonen J, Laitinen MP, Vuojolainen K, et al. Human growth differentiation factor 9 (GDF-9) and its novel homolog GDF-9B are expressed in oocytes during early folliculogenesis. J Clin Endocrinol Metab 1999;84:2744-50.

45. Teixeira Filho FL, Baracat EC, Lee TH, et al. Aberrant expression of growth differentiation factor-9 in oocytes of women with polycystic ovary syndrome. J Clin Endocrinol Metab 2002 87:1337-44.

46. Sadeu JC, Smitz J. Growth differentiation factor-9 and anti-Mullerian hormone expression in cultured human follicles from frozen-thawed ovarian tissue. Reprod Biomed Online 2008;17:537-48.

47. Hayashi M, McGee EA, Min G, et al. Recombinant growth differentiation factor-9 (GDF-9) enhances growth and differentiation of cultured early ovarian follicles. Endocrinology 1999;140:1236-44.

48. Hreinsson JG, Scott JE, Rasmussen C, et al. Growth differentiation factor-9 promotes the growth, development, and survival of human ovarian follicles in organ culture. J

Cite this article as: $\mathrm{Wu} \mathrm{Y,} \mathrm{Sun} \mathrm{Z,} \mathrm{Wang} \mathrm{Y,} \mathrm{Chen} \mathrm{H,} \mathrm{Bian} \mathrm{J.}$ Human dermal fibroblasts support the development of human primordial/primary follicles in a 3-dimensional alginate matrix culture system. Ann Transl Med 2021;9(10):868. doi: 10.21037/ atm-21-2125
Clin Endocrinol Metab 2002;87:316-21.

49. Nilsson EE, Skinner MK. Growth and differentiation factor-9 stimulates progression of early primary but not primordial rat ovarian follicle development. Biol Reprod 2002;67:1018-24.

50. Wang J, Roy SK. Growth differentiation factor-9 and stem cell factor promote primordial follicle formation in the hamster: modulation by follicle-stimulating hormone. Biol Reprod 2004;70:577-85.

51. Otsuka F, Yao Z, Lee T, et al. Bone morphogenetic protein-15. Identification of target cells and biological functions. J Biol Chem 2000;275:39523-528.

52. Wei LN, Liang XY, Fang C, et al. Abnormal expression of growth differentiation factor 9 and bone morphogenetic protein 15 in stimulated oocytes during maturation from women with polycystic ovary syndrom. Fertil Steril 2011;96:464-68.

53. Unger C, Gao S, Cohen M, et al. Immortalized human skin fibroblast feeder cells support growth and maintenance of both human embryonic and induced pluripotent stem cells. Hum Reprod 2009;24:2567-81.

(English Language Editor: J. Reynolds) 